NASA/CR-97-206251

ICASE Report No. 97-64

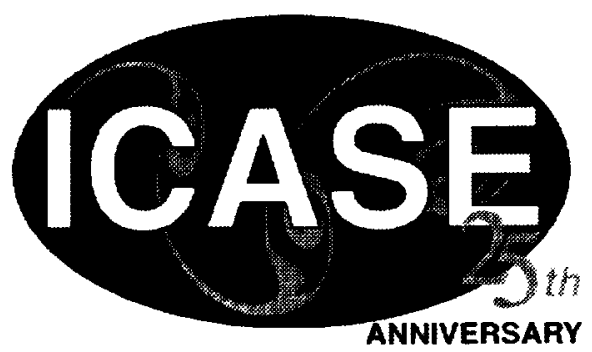

\title{
On the Universality of the Kolmogorov Constant in Numerical Simulations of Turbulence
}

P. K. Yeung

Georgia Institute of Technology

Ye Zhou

ICASE and IBM

Institute for Computer Applications in Science and Engineering

NASA Langley Research Center

Hampton, VA

Operated by Universities Space Research Association

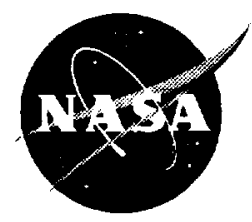

National Aeronautics and

Space Administration

Langley Research Center

Hampton, Virginia 23681-2199 
Available from the following:

NASA Center for AeroSpace Information (CASI) 800 Elkridge Landing Road

Linthicum Heights, MD 21090-2934

(301) 621-0390
National Technical Information Service (NTIS)

5285 Port Royal Road

Springfield, VA 22161-2171

(703) $487-4650$ 


\title{
ON THE UNIVERSALITY OF THE KOLMOGOROV CONSTANT IN NUMERICAL SIMULATIONS OF TURBULENCE*
}

\author{
P.K.YEUNG ${ }^{\dagger}$ AND YE ZHOU ${ }^{\ddagger}$
}

\begin{abstract}
Motivated by a recent survey of experimental data [K.R. Sreenivasan, Phys. Fluids 7, 2778 (1995)], we examine data on the Kolmogorov spectrum constant in numerical simulations of isotropic turbulence, using results both from previous studies and from new direct numerical simulations over a range of Reynolds numbers (up to 240 on the Taylor scale) at grid resolutions up to $512^{3}$. It is noted that in addition to $k^{-5 / 3}$ scaling, identification of a true inertial range requires spectral isotropy in the same wavenumber range. We found that a plateau in the compensated three-dimensional energy spectrum at $k \eta \approx 0.1--0.2$, commonly used to infer the Kolmogorov constant from the compensated three-dimensional energy spectrum, actually does not represent proper inertial range behavior. Rather, a proper, if still approximate, inertial range emerges at $k \eta \approx 0.02-0.05$ when $R_{\lambda}$ increases beyond 140 . The new simulations indicate proportionality constants $C_{1}$ and $C$ in the one- and three-dimensional energy spectra respectively about 0.60 and 1.62. If the turbulence were perfectly isotropic then use of isotropy relations in wavenumber space $\left(C_{1}=18 / 55 C\right)$ would imply that $C_{1} \approx 0.53$ for $C=1.62$, in excellent agreement with experiments. However the one- and three-dimensional estimates are not fully consistent, because of departures (due to numerical and statistical limitations) from isotropy of the computed spectra at low wavenumbers. The inertial scaling of structure functions in physical space is briefly addressed. Since DNS is still restricted to moderate Reynolds numbers, an accurate evaluation of the Kolmogorov constant is very difficult. We focus on providing new insights on the interpretation of Kolmogorov 1941 similarity in the DNS literature and do not consider issues pertaining to the refined similarity hypotheses of Kolmogorov (K62).
\end{abstract}

Key words. Kolmogorov constant, energy spectrum, numerical simulations

Subject classification. Fluid Mechanics

1. Introduction. Inertial-range behavior as postulated by Kolmogorov's (K41) similarity hypotheses [1] is widely regarded as a fundamental characteristic of turbulence at high Reynolds number. In particular, in the inertial range of intermediate scales the K41 result for the one-dimensional longitudinal energy spectrum is given by

$$
E_{11}\left(k_{1}\right)=C_{1}\langle\epsilon\rangle^{2 / 3} k_{1}^{-5 / 3}
$$

where $k_{1}$ is the longitudinal wavenumber, $C_{1}$ is known as the Kolmogorov constant, and $\langle\epsilon\rangle$ is the mean dissipation rate. Although $\mathrm{K} 41$ theory is subject to intermittency corrections associated with dissipation rate fluctuations, such effects are primarily manifested in higher-order statistics. Indeed, intermittency effects on the second-order energy spectrum exponent are believed to be small and hardly measurable (Kolmogorov [2], Frisch [3]), while at the same time may contribute to a persistence Reynolds number dependence for higher-order structure functions (L'vov \& Procaccia [4]).

*This research was supported in part by NSF Grant No. CTS-930 7973 to the first author, and by NASA under Contract No. NAS1-19480 while the second author was in residence at ICASE.

${ }^{\dagger}$ School of Aerospace Engineering, Georgia Institute of Technology, Atlanta, GA 30332.

$\ddagger$ Institute for Computer Applications in Science and Engineering, NASA Langley Research Center, Hampton, VA 23681 and IBM Research Division, T.J. Watson Research Center, P.O. Box, 218, Yorktown Heights, NY 10598. 
The classical view of the "five-thirds" scaling law above, with substantial experimental support (e.g., Monin \& Yaglom [5], Sec. 23.3), is that $C_{1}$ has an universal value at asymptotically high Reynolds number. Recently, however, there is renewed debate on the universality of $C_{1}$, in part because of new measurements at high Reynolds number (Praskovsky \& Oncley [6]) and a subsequent new similarity theory (Barenblatt \& Goldenfeld [7]) that suggested a persistent Reynolds number dependence even at high Reynolds numbers. On the other hand, the conclusion from a new and very extensive survey of experimental data by Sreenivasan [8] is that, taken collectively, measurements do not support such a dependence for the Kolmogorov constant. Sreenivasan found that the value of $C_{1}$ averaged over the many different experiments cited is about $0.53 \pm 0.055$, although some corrections for the estimation of dissipation using local isotropy assumptions in experiments may be warranted. It is still possible (Barenblatt \& Goldenfeld [7], Sreenivasan [8]), though, that controlled experiments for a single geometry over a wide range of Reynolds numbers may reveal signifcant trends otherwise masked by experimental scatter; new measurements of this nature (suggesting strong Reynolds number dependence) have been reported by Mydlarski \& Warhaft [9].

This paper is motivated by the survey of experimental data noted above, and will focus on similar issues arising in numerical simulations of isotropic turbulence. We first review the basis for estimating the Kolmogorov constant from previous studies, and then present new results from direct numerical simulations (DNS) over a range of Reynolds numbers. Because of Reynolds number considerations, an accurate evaluation of the Kolmogorov constant by DNS is admittedly very difficult. As such, we limit ourselves to the task of providing new insights on the interpretation of K41 similarity in the DNS literature. For similar reasons, we shall not consider the use of DNS to study issues pertaining to the refined similarity hypotheses of Kolmogorov (K62) [2] (see, e.g., Chen et al. [10], Wang et al. [11] and others).

In numerical simulations, $\mathrm{K} 41$ similarity is frequently discussed in terms of the three-dimensional energy spectrum function $E(k)$ (where $k$ is the wavenumber magnitude). If the turbulence at scale size $1 / k$ is isotropic then a kincmatic constraint relating one- and three-dimensional spectra is

$$
E(k)=\frac{1}{2} k^{3} \frac{d}{d k}\left(\frac{1}{k} \frac{d E_{11}(k)}{d k}\right) .
$$

Substitution of Eq. 1 into Eq. 2 implies that in the inertial range

$$
E(k)=C\langle\epsilon\rangle^{2 / 3} k^{-5 / 3},
$$

where $C=55 / 18 C_{1}$. In principle, therefore, one may obtain either $C$ or $C_{1}$ (from three- and one-dimensional spectra respectively) and infer the other using isotropy relations. Values of the Kolmogorov constant $C$ (estimated using either of these approaches) cited in a number of recent high resolution numerical studies by other authors [11-21] are collected in Table I. Some relevant results from large-eddy simulations (LES) are included also. (Note that we have quoted values of $C$ from the highest-resolution data in each of these references, since from the viewpoint of present paper we should not infer the Kolmogorov constant from low-resolution simulations, especially in the older data.)

It may be noticed that most of the values displayed in Table I are higher than the value 1.619 , which is $55 / 18$ of the experimental average of 0.53 for $C_{1}$. Note that, however, Zhou [14] found that the Kolmogorov constant $C=1.5$ from calculations of the spectra energy flux based on the self-similarity condition of the triadic energy transfer function, in the form $T(k, p, q)=a^{3} T(a k, a p, a q)$ (where $a$ is a scaling constant). A commonly used procedure (e.g. Kerr [17], Jiménez et al. [20]) for estimating the value of $C$ is to plot the "compensated" three-dimensional energy spectrum

$$
\psi(k) \equiv E(k)\langle\epsilon\rangle^{-2 / 3} k^{5 / 3}
$$


as a function of wavenumber normalized by the Kolmogorov scale $(\eta)$, and to interpret $C$ as the height of a "plateau". However, it should be noted that whereas a flat region in the compensated spectrum implies $k^{-5 / 3}$ behavior, the observation of a $k^{-5 / 3}$ scaling range is not by itself a sufficient condition for an inertial range. It is important that isotropy also be attained in the wavenumber range which displays $k^{-5 / 3}$ behavior. (If the isotropy requirement were relaxed then in principle one would have a different value of the "Kolmogorov constant" in each direction, and the concept of universality would be lost.) Indeed, as will be seen in the rest of this paper, our new results demonstrate that deviations from isotropy can contribute to values of $C$ that appear to be too high.

The major limitation in using DNS to examine inertial-range dynamics is, of course, the difficulty in attaining high Reynolds numbers. However, recent advances in massively parallel computing have shown significant promise. Our simulations were performed using a parallel implementation [22] of the well-known Fourier pseudo-spectral algorithm of Rogallo [23] on the IBM SP at the Cornell Theory Center. The highest grid resolution used is $512^{3}$, with a Taylor-scale Reynolds number $\left(R_{\lambda}\right)$ about 240 averaged over about four eddy-turnover times. Whereas this Reynolds number is not high compared to some recent laboratory experiments ( $R_{\lambda} 473$ in Mydlarski \& Warhaft [9]), it is about the same as (or slightly higher than) the highest values reported in the DNS literature (for example, $R_{\lambda} 218$ in Cao et al. [24]). Numerical results on spectra as well as structure functions (to which Kolmogorov [1] originally referred) are given in the next section. In order to characterize issues of Reynolds number dependence and attempt to quantify a minimum Reynolds number threshold above which inertial-range behavior could be expected, we present data at five different grid resolutions.

To maintain a stationary state so that results may be averaged over time at the highest Reynolds number possible using a given number of grid points, it is usual to apply numerical forcing at the large scales. In the literature this has been done in several different ways, such as adding a stochastic forcing term (Eswaran \& Pope [25]), holding the energy in the lowest-wavenumber shells fixed while allowing phase information to evolve (Chen et al. [10], Sullivan et al. [26]), or by introducing a negative viscosity in these shells (Jiménez et al. [20]). Alternatively, LES [12-15], with and without forcing, as well as simulations with a hyperviscosity [16] can also be used to achieve higher Reynolds numbers. However, it is generally believed that (c.g., see Ref. 20), the precise manner of forcing--provided it is applied to the largest scales in the flow-has no systematic effects on the inertial-range energy spectrum, nor on the statistical character of the small scales. Whereas in this work we have used the scheme of Eswaran \& Pope [25], we have checked that similar calculations in which the energy in the first couple of wavenumber shells is fixed do not warrant different conclusions.

2. Results. We present spectra and structure functions from simulations of forced stationary isotropic turbulence averaged over relatively long time periods. Major simulation parameters are summarized in Table II. For the less expensive $64^{3}$ and $128^{3}$ runs the averaging time $(T)$ shown is the aggregate of multiple simulations of shorter duration. In all cases the small scales are considered to be well resolved, as measured by $k_{\max } \eta \approx 1.5$, where $k_{\max }$ is the highest wavenumber represented by the grid points. The non-dimensional quantity $\langle\epsilon\rangle L_{1} / u^{\prime 3}$ (where $L_{1}$ is the longitudinal integral length scale derived from the longitudinal velocity correlation, and $u^{\prime}$ is the r.m.s. velocity) in the last column of this table is of interest in the scaling of $\langle\epsilon\rangle$ with $u^{\prime 3} / L_{1}$ using energy cascade arguments; it appears to approach a constant at high Reynolds numbers. This trend is similar to that found in the simulations of Jiménez et al. [20], Wang et al. [11] and Cao et al. [24], and to that in experiments (Sreenivasan [27], Fig. 1). The differences among different "asymptotic" numerical values are due in part to differences in the definition of integral scales and in flow conditions in 
experiments and numerical simulations.

Figure 1 shows the three-dimensional compensated spectrum $\psi(k)$ in Kolmogorov scaling. (Note: in these and all other figures lines $A-E$ refer to data at five different grid resolutions from $64^{3}$ to $512^{3}$ respectively, as listed in Table II.) Except for small spectral turn-ups at the high wavenumber end resulting from residual aliasing errors, small-scale universality is unambiguously achieved, even at the lowest Reynolds number $\left(R_{\lambda} 38\right.$ in $64^{3}$ simulations). Two relatively flat (i.e. $\left.k^{-5 / 3}\right)$ regimes of limited extent can be seen: namely at $k \eta \approx 0.1-0.2$ in the form of a bumpy "plateau" which is seen at all grid resolutions, and in the low-wavenumber range $k \eta \approx 0.02-0.05$ which is captured only at higher (256 ${ }^{3}$ and beyond) resolutions. If the former were taken to represent the inertial range then one would obtain a Kolmogorov constant $C$ greater than 2.0, as reported by a number of other authors (see Table I). On the other hand, it is clear that the level of $\psi(k)$ in the range $k \eta \approx 0.02-0.05$ agrees well with experimental data, if one uses the isotropy relation $C=55 / 18 C_{1}$, which would imply $C_{1}=18 / 55 C \approx 0.53$. It is our objective in the analyses below to establish that this lower-wavenumber region, rather than the plateau, represents (the beginnings of) a proper inertial range.

In order that inertial range dynamics can be independent of both the large-scale energetics and viscous dissipation, a wide scale separation must exist between the peaks of the energy and dissipation spectra in wavenumber space. The peak of $E(k)$ occurs in the lowest two wavenumber shells $\left(k \eta \approx 0.01\right.$ for the $512^{3}$ data), whereas from a plot of the dissipation spectrum we find that the peak of $D(k) \equiv 2 \nu k^{2} E(k)$ occurs at $k \eta \approx 0.17$. This location of the dissipation spectral peak is virtually the same as found by Wang et al. [11]. Since this almost coincides with the "plateau" in Fig. 1 it is clear that the plateau should not be taken as an indication of inertial-range behavior. In fact, this plateau may be identified with the so-called "bottleneck" phenomenon that has been discussed in experimental (Saddoughi \& Veeravalli [28]), theoretical (Falkovich [29]), and numerical (Borue \& Orszag [30]) work. According to this theory, viscous effects on spectral transfer causes an increase (relative to $k^{-5 / 3}$ in the energy spectrum at intermediate wavenumbers. Nonlocal interactions among Fourier modes well separated in scale (Brasseur \& Wei [31]) are also believed to play an important role (Herring et al. [32]). We also note that Brasseur \& Wei argued that there should be about a decade in scale separation between the wavenumber where the inertial range "ends" and the dissipation peak, which is more than that (about 3 to 4) found in DNS (Wang et al. [11] and this paper). A full understanding of this latter issue awaits future high-resolution simulations and experimental data.

A more direct comparison with experiment may be made by considering the compensated longitudinal energy spectrum, i.e. $\psi_{1}\left(k_{1}\right)=E_{11}\left(k_{1}\right)\langle\epsilon\rangle^{-2 / 3} k_{1}^{5 / 3}$, which is shown in Fig. 2. Because this spectrum drops off very rapidly at high wavenumbers, we have used log-linear scales to highlight the function values at any wavenumber ranges where $\psi_{1}$ is approximately constant. Two such ranges can be seen, corresponding to those for $\psi$ but in each case occurring at lower wavenumbers. The observation that the peak of $\psi_{1}\left(k_{1}\right)$ occurs at $k_{1} \eta=0.05$ is similar to high Reynolds number measurements in both boundary layers (Saddoughi \& Veeravalli [28]) and grid turbulence (Mydlarski \& Warhaft [9]). It is also apparent that the value of $C_{1}$ inferred from this figure is about 0.60 , which is somewhat higher than, but still relatively close to the experimental average [5] of 0.53 .

From Figs. 1 and 2 we may conclude that the best values obtained for $C_{1}$ from the three- and onedimensional spectra are about 0.53 and 0.60 respectively. These values (especially the former) are closer to experiment than those cited previously in the literature. Yet there is evidently some inconsistency between these two estimates for $C_{1}$. These differences are due to deviations from isotropy in wavenumber space, as further studied below. 
In addition to $E_{11}\left(k_{1}\right)$ we have also computed the transverse energy spectrum, for which the classical inertial-range result is

$$
E_{22}\left(k_{1}\right)=C_{1}^{\prime}\langle\epsilon)^{2 / 3} k_{1}^{-5 / 3}
$$

where isotropy requires $C_{1}^{\prime}=\frac{4}{3} C_{1}$. The compensated transverse spectrum (Fig. 3) exhibits a similar but stronger bump at $k_{1} \eta \approx 0.07$. We also find that the ratio $E_{22}\left(k_{1}\right) / E_{11}\left(k_{1}\right)$ is close to $4 / 3$ in the range $k_{1} \eta \approx 0.02-0.04$, but increases steadily with wavenumber beyond this range.

If a five-thirds scaling holds for both one- and threc-dimensional spectra over the same wavenumber range then the functions $E(k)$ and $E_{11}(k)$ should be proportional within this range. Figure 4 shows the spectral ratio $E(k) / E_{11}(k)$, compared with the classical inertial range value of $55 / 18$. It may be seen that, at the wavenumber range $(k \eta \approx 0.1-0.2)$ corresponding to the plateau in Fig. 1, this ratio is considerably higher than 55/18 and in fact increases roughly in proportion to $k \eta$. This observation provides further evidence that the plateau in $\psi$ does not represent an inertial range. The transition from behavior without a level region to one at a value close to $55 / 18$ (at $R_{\lambda} 240$ at $512^{3}$ resolution) occurs between $R_{\lambda} 90$ (on an $128^{3}$ grid) and $R_{\lambda} 140$ (for $256^{3}$ ). The ratio $C / C_{1}$ inferred in this manner is seen to be somewhat less than $55 / 18$.

It should be noted that isotropy of the spectral tensor is a key requirement for the relation $C=55 / 18 C_{1}$. Although the simulations are of (nominally) isotropic turbulence, departures of the computed spectra from isotropy are, in fact, not totally unexpected. It is well-known that sampling limitations arise in the lowest few wavenumber shells because relatively few samples of the large scales are present in a solution domain of finite size. Furthermore, since the solution domain in physical space is a cube (rather than a sphere), the averaged statistics are at best invariant among the three Cartesian coordinate axes, but do not satisfy the stricter requirement of no preferential orientation in three-dimensional space. As an example we may note that whereas the periodicity length of the flow along the coordinate axes is equal to the length of each side of the solution domain, it is longer if measured along directions inclined to the axes. This effect is felt primarily in spatial correlations over large separations in space, which correspond to low wavenumbers in Fourier space.

In Figs. 6 and 7 these structure functions are shown in Kolmogorov scaling in order to compare with the theoretical proportionality constants $C_{2} \approx 4.02 C_{1}$ and $-4 / 5$. It is clear that the highest Reynolds number data shown agree well with classical inertial range results. Numerical values of $-D_{L L L}(r) /\langle\epsilon\rangle r$ at intermediate resolutions also fit in well within the Reynolds number trend suggested by new measurements in grid turbulence over a range of Reynolds numbers (Sreenivasan \& Dhruva [35]).

It is perhaps worth noting that the highest-resolution $\left(512^{3}\right)$ simulation reported is at a higher Reynolds number and are averaged over a greater number of large-eddy turnover times than several other studies reporting $512^{3}$ results (e.g., Jiméncz et al. [20], Chen et al. [10], Wang et al. [11]) for stationary isotropic turbulence. The spectra and structure functions obtained from these and the present simulations demonstrate that, with the latest advances in massively parallel computing, issues concerning inertial-range similarity in DNS can now be addressed in a more reliable manner than possible before.

3. Conclusions . We have presented new results on the Kolmogorov scaling of energy spectra and structure functions in the inertial range, from direct numerical simulations of stationary isotropic turbulence ranging from $R_{\lambda} 38$ (on a $64^{3}$ grid) to $R_{\lambda} 240$ (on $512^{3}$ ). It is pointed out that a plateau in the compensated three-dimensional energy spectrum at $k \eta \approx 0.1-0.2$ commonly used to infer the Kolmogorov constant from the compensated three-dimensional energy spectrum in fact does not represent proper inertial range 
behavior. Instead, a proper (if still approximate) inertial range emerges at $k \eta \approx 0.02-0.05$ when $R_{\lambda}$ increases beyond 140 . We find that the proportionality constants $C$ and $C_{1}$ in the three- and one-dimensional compensated energy spectra are about 1.62 and 0.60 respectively. These values are closer to experimental data than reported in most previous numerical simulations. In particular, if isotropy relations are used then we may infer from the three-dimensional spectra that $C_{1}=18 / 55 C \approx 0.53$, in excellent agreement with experimental data (Sreenivasan [8]). However, the ratio $C / C_{1}$ in our results differs from the theoretical value of $55 / 18$, because of significant departures from isotropy in the computed spectra at the wavenumber range where inertial-range behavior is otherwise reasonably well approximated. Results on second- and third-order structure functions over a range of Reynolds numbers further suggest that the simulation database that we have accumulated should be useful for investigating other aspects of similarity scaling and Reynolds number dependence.

We emphasize that a $k^{-5 / 3}$ scaling is in itself not a sufficient indicator of inertial-range behavior. To achieve a strictly isotropic inertial range in direct numerical simulations requires that the inertial scales be small compared to the size of the solution domain. Whereas it is difficult to meet this requirement well, it seems clear that high-resolution simulations using the techniques of massively parallel computing are very helpful.

Acknowledgments. We are grateful to Professor K.R. Sreenivasan for many stimulating discussions and his constant encouragement, as well as comments on an early draft of this paper. In addition we thank Professors Jim Brasseur, Zellman Warhaft, Drs. Shiyi Chen and Jack Herring for their valuable input. Figures 6 and 7 evolved from joint work between the first author and Dr. Michael Borgas, within NSF Grant INT-9526868. The computations were performed using the supercomputing resources of the Cornell Theory Center, which receives major funding from NSF and New York State.

\section{REFERENCES}

[1] Kolmogorov, A.N., 1941. The local structure of turbulence in incompressible viscous fiuids for very large Reynolds numbers, Dokl. Akad. Nauk SSSR, vol 30, p. 301.

[2] Kolmogorov, A.N., 1962. 'A refinement of previous hypotheses concerning the local structure of turbulence in a viscous incompressible fluid at high Reynolds number, J. Fluid Mech. vol. 13, p. 82.

[3] Frisch, U., 1995. Turbulence: The Legacy of A.N. Kolmogorov, Cambridge University Press.

[4] L'vov, V.S., AND PROCACCIA, I., 1995. Intermittency in hydrodynamic turbulence as intermediate asymptotics to Kolmogorov scaling, Phys. Rev. Lett. vol. 74, p. 2690.

[5] Monin, A.S, ANd Yaglom, A.M., 1975. Statistical Fluid Mechanics, Vol. II, MIT Press.

[6] Praskovsky, A., AND Oncley, S., 1994. Measurements of the Kolmogorov constant and intermittency exponent at very high Reynolds numbers, Phys. Fluids, vol. 6, 2886.

[7] Barenblatt, G.I., AND Goldenfeld, N., 1995. Does fully developed turbulence exist? Reynolds number independence versus asymptotic covariance, Phys. Fluids, vol. 7, 3078. bitem

[8] SReentvasan, K.R., 1995. On the universality of the Kolmogorov constant, Phys. Fluids, p. 2778.

[9] MYDLARSKI, L., AND WARHAFT, Z., On the onset of high-Reynolds-number grid-generated wind tunnel turbulence, J. Fluid Mech., vol. 320, 331.

[10] Chen, S., Doolen, G.D., Kraichnan, R.H., and She, Z.-S., 1993. On statistical correlations between velocity increments and locally averaged dissipation in homogeneous turbulence, Phys. Fluids A, vol. 5, 458 . 
[11] Wang, L.P., Chen, S., Brasseur, J.G., And WyngaARd, J.C., 1996. Examination of hypotheses in the Kolmogorov refined turbulence theory through high-resolution simulations: Part 1. Velocity field, J. Fluid Mech., vol. 309, 113.

[12] LesieUR, M. \& Rogallo, R.S., 1989. Large-eddy simulation of passive scalar diffusion in isotropic turbulence, Phys. Fluids A, vol. 1, p.718.

[13] Chasnov, J.R., 1991. Simulation of the Kolmogorov inertial range using an improved subgrid model, Phys. Fluids A, vol. 1, p.945.

[14] ZноU, Y., 1993. Interacting scales and energy transfer in isotropic turbulence, Phys. Fluids A, vol. 5, p.2524.

[15] ShE, Z.-S., ANd JACKson, E., 1993. A constrained Euler system for Navier-Stokes turbulence, Phys. Rev. Lett., vol. 70, 1255.

[16] Borue, V., ANd Orszag, S.A., 1995. Self-similar decay of three-dimensional homogeneous turbulence with hyperviscosity, Phys. Rev. E, vol. 51, p. R856.

[17] KERR, R.M., 1990. Velocity, scalar, and transfer spectra in numerical turbulence J. Fluid Mech., vol. 211, p.309.

[18] Vincent, A., ANd Meneguzzi, M., 1991. The spatial structure and statistical properties of homogeneous turbulence, J. Fluid Mech., vol. 225, p. 1.

[19] SANAdA, T., 1992. Comment on the dissipation-range spectrum in turbulent flows, Phys. Fluids A, vol. 4, p. 1086.

[20] Jiménez, J., Wray, A.A., Saffman, P.G., And Rogallo, R.S., 1993. The structure of intense vorticity in isotropic turbulence, J. Fluid Mech., vol. 255, 65.

[21] Hosokawa, I., OIDE, S., AND Yamamoto, K., 1996. Isotropic turbulence: important differences between true dissipation rate and its one-dimensional surrogate, Phys. Rev. Lett., vol. 77, 4548. .

[22] Yeung, P.K., And Moseley, C., 1995. in Parallel Computational Fluid Dynamics: Implementations and Results Using Parallel Computers., eds. A. Ecer, J. Periaux, N. Satofuka \& S. Taylor (Elsevier, Amsterdam), 473.

[23] Rogallo, R.S., 1981. Numerical experiments in homogeneous turbulence, NASA Tech. Memo. 81315.

[24] CAO, N., Chen, S., ANd Doolen, G., 1996. Statistics and structure of pressure in isotropic turbulence", Submitted to Phys. Fluids.

[25] Eswaran, V., AND POPE, S.B., An examination of forcing in direct numerical simulations of turbulence, Comput. \& Fluids, vol. 16, p. 257.

[26] Sullivan, N.P., Mahalingham, S., AND KerR, R.M., 1994. Deterministic forcing of homogeneous, isotropic turbulence, Phys. Fluids vol. 6, 1612.

[27] SReEnivasan, K.R., 1984. On the scaling of the turbulence energy dissipation rate, Phys. Fluids vol. 27, p.1048.

[28] Saddoughi, S.G., ANd Veeravalli, S.V., 1994. Local isotropy in turbulent boundary layers at high Reynolds number, J. Fluid Mech., vol. 268, p. 333.

[29] Falkovich, G., 1994. Bottleneck phenomenon in developed turbulence, Phys. Fluids vol. 6, p. 1411.

[30] Borue, V., AND Orszag, S.A., 1996. Numerical study of three-dimensional Kolmogorov flow at high Reynolds numbers, J. Fluid Mech. vol. 306, p. 293.

[31] BrasseuR, J.G., AND WEI, C.H., 1994. Interscale dynamics and local isotropy in high Reynolds number turbulence within triadic interactions, Phys. Fluids vol. 6, p. 842.

[32] Herring, J.R., Schertzer, D., Lesieur, M., Newman, G.R., Chollet, J.P., And 
LARChEVEQUE, M., 19823. A comparative assessment of spectral closure as applied to passive scalar diffusion, J. Fluid Mech. vol. 124, p. 411.

[33] Yeung, P.K., Brasseur, J.G., AND WANG, Q., 1995. Dynamics of direct large-small scale couplings in coherently forced turbulence: Concurrent physical and Fourier-space view, J. Fluid Mech. vol. 283, p. 43.

[34] Sreenivasan, K.R., and Dhruva, B., 1996. private communication.

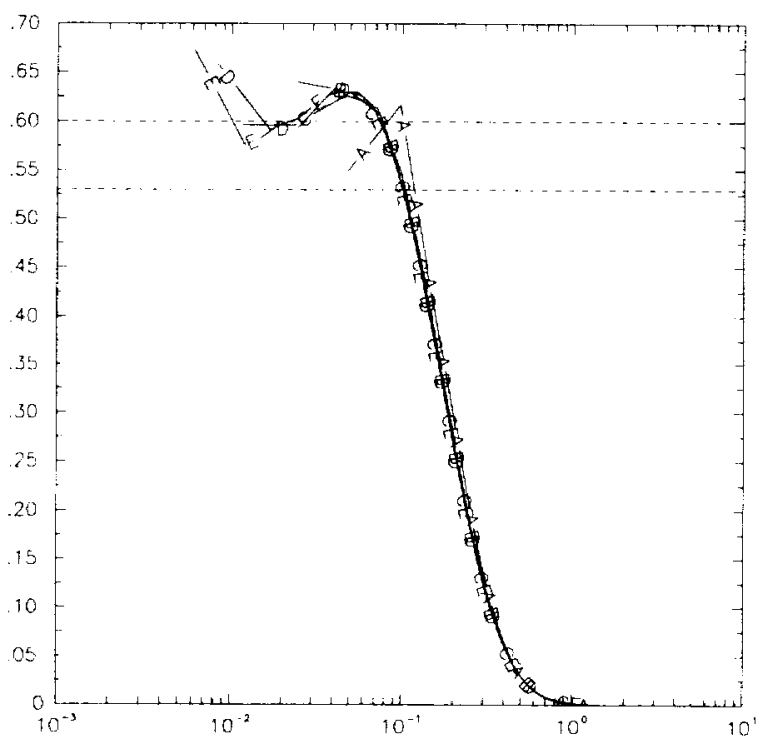

FIG. 1. Compensated three-dimensional energy spectrum $\psi(k)$ versus Kolmogorov-scaled wavenumber kn, at five different Reynolds numbers and grid resolutions (see Table II). Dashed horizontal lines at levels $1.619=(55 / 18) 0.53$ and 2.0 are drawn for reference. 
TABLE 1

Kolmogorov constant computed from DNS and LES ${ }^{a}$

authors method Grid $R_{\lambda} \quad C(1 \mathrm{D})^{b} \quad C(3 \mathrm{D})$

Lesieur\&Rogallo [12] Forced LES ${ }^{c} \quad 128^{3} \quad$ - $1.5-1.8$

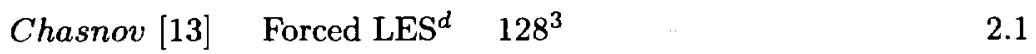

$\begin{array}{llllll}\text { Zhou }[14] & \text { LES }^{e} & 256^{3} & - & - & 1.5\end{array}$

$\begin{array}{lllll}\text { She\&Jackson }[15] & \operatorname{LES}^{f} & 128^{3} & - & 1.88\end{array}$

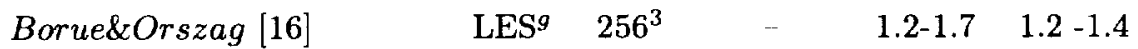

$\begin{array}{lllll}K e r r[17] & \text { Forced DNS } & 128^{3} & 82 & 2\end{array}$

Vincent\&Meneguzzi[18] Forced DNS $240^{3} \quad 150 \quad 2$

Sanda[19] Forced DNS $256^{3} \quad 120 \quad \cdots \quad 2$

Jimenez et al[20] Forced DNS $512^{3} \quad 170-\quad 0.91-1.22 \quad 2$.

Wang et al.[11] $\quad$ Forced DNS ${ }^{h} \quad 512^{3} \quad 190 \quad 1.68 \quad 1.5-2$.

$\begin{array}{lllll}\text { Hosokawa et al. [21] } & \text { DNS } & 512^{3} & 160 & 2.1\end{array}$

\section{Footnotes}

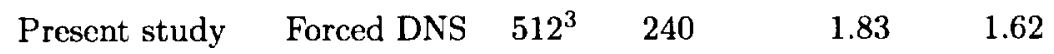

a We included most of the recent numerical data on the Kolmogorov constant.

${ }^{b} C$ (1D) is obtained as $(55 / 18) C_{1}$, assuming isotropy in the inertial range. (Hosokawa et al. used $0.76 C_{2}$, from the structure function.)

$c$ Spectral LES with a spectral eddy viscosity.

${ }^{d}$ Spectral LES with an eddy viscosity and a stochastic force.

e Zhou used simulation databases from a constrained energy simulation where a "five-thirds" spectrum is maintained. Furthermore, the self-similarity condition is used to compute an ideal Kolmogorov energy transfer function. The Kolmogorov constant is determined from the flux.

$f$ She and Jackson used the constrained Euler system simulation. The Kolmogorov constant is determined from the flux.

$g$ Borue and Orszag reported their decaying DNS at $256^{3}$ resolution along with a hyperviscosity. Both $C$ (1D) and $C$ (3D) are estimated by the authors of present paper from Fig. 4 of Ref. 16. The contributions from the "bumps" are ignored.

${ }^{h}$ Wang et al. argued that the Kolmogorov constant is more accurate when estimated from $C$ (1D) followed by using the isotropy relation. 
TABLE 2

Major simulation parameters in the new results.

$\begin{array}{lrrr}\text { Grid } & R_{\lambda} & T / T_{E} & \langle\epsilon\rangle L_{1} / u^{\prime 3} \\ 64^{3} & 38 & 146 & 0.693 \\ 128^{3} & 90 & 139 & 0.499 \\ 256^{3} & 140 & 9.8 & 0.475 \\ 384^{3} & 180 & 3.2 & 0.419 \\ 512^{3} & 240 & 3.9 & 0.416\end{array}$

Note: $T$ is the total averaging time period, and $T_{E}$ is the eddy-turnover time (the ratio of the longitudinal integral length scale, $L_{1}$, to the r.m.s. velocity fluctuation, $u^{\prime}$ ) 


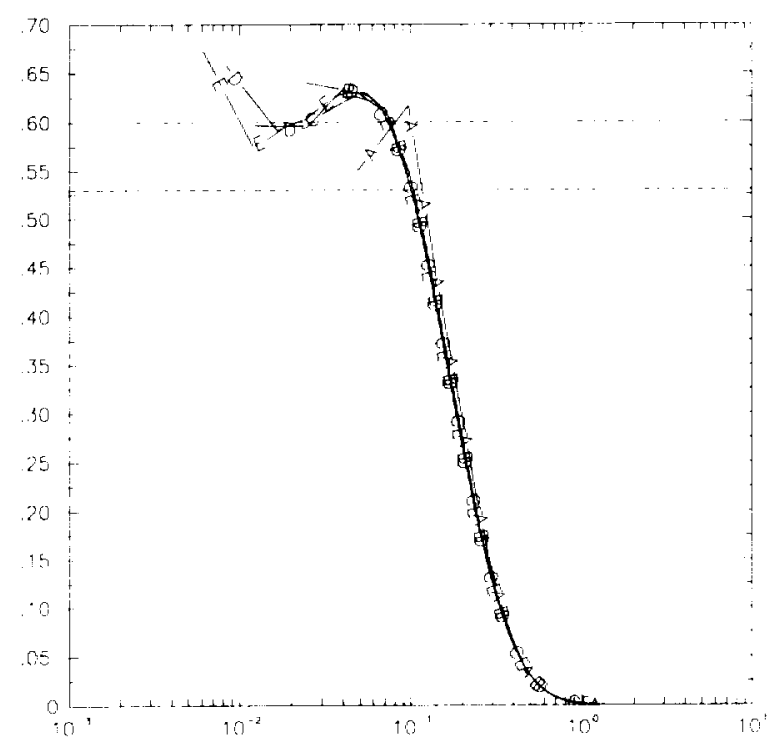

FIG. 2. Compensated one-dimensional energy spectrum versus Kolmogorov-scaled wavenumber $k_{1} \eta$, at five different Reynolds numbers and grid resolutions (see Table II). The reference lines are drawn at levels 0.53 and 0.60 .

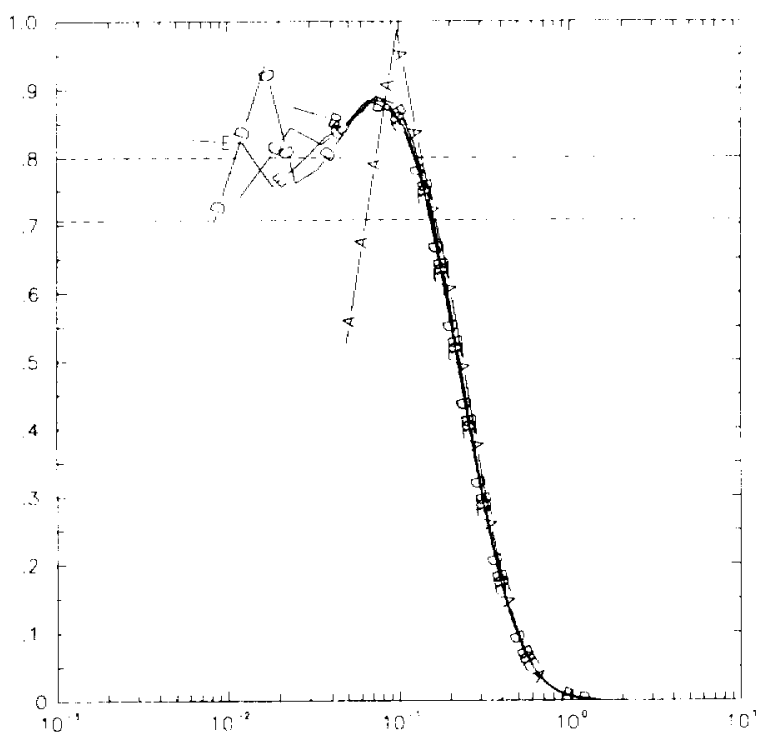

Fig. 3. Same as Fig. 2, but for the transverse spectrum. The reference lines are drawn at levels $0.707=(4 / 3) 0.53$ and 0.8 .

11 


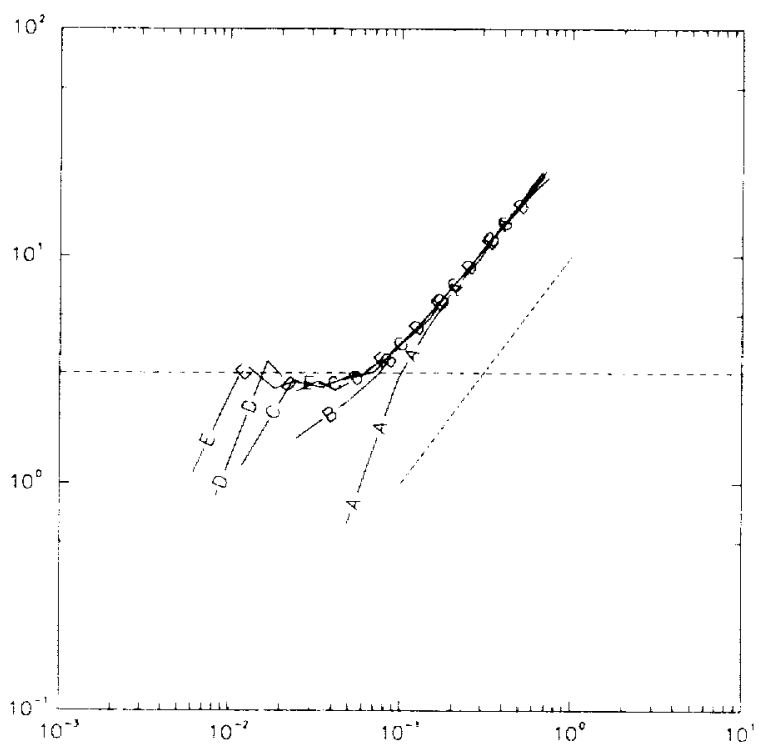

Fig. 4. The ratio $E(k) / E_{11}(k)$ versus Kolmogorov-scaled wavenumber. The horizontal line is at $55 / 18$, whereas the sloping line has slope unity. Because this ratio attains very large values at high wavenumbers, only the lower half of the wavenumber range in each simulation is shown.

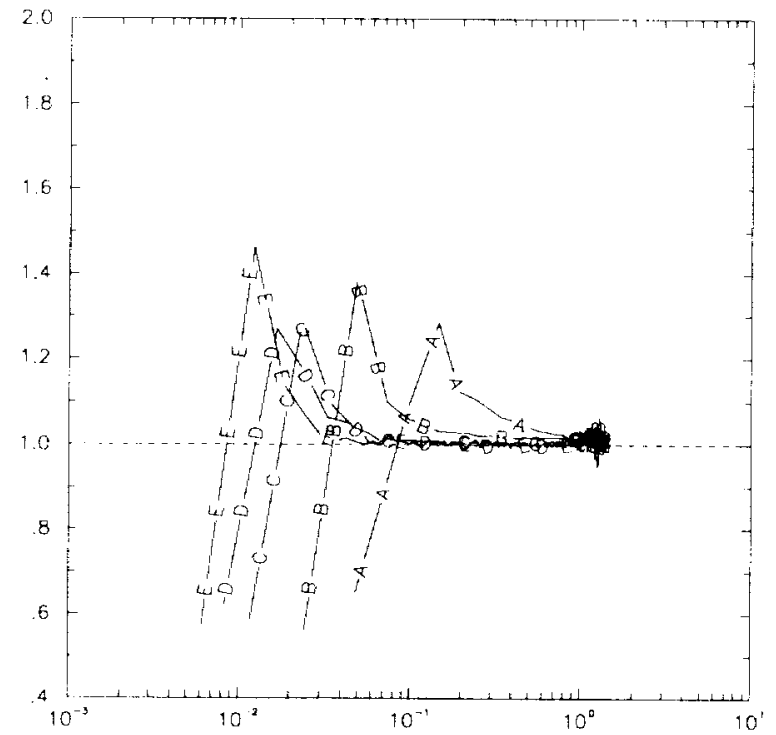

FIG. 5. The isotropy coefficient $I\left(k_{1}\right)$ (see Eq. 6) versus Kolmogonov-scaled one-dimensional wavenumber. (A value of unity signifies spectral isotropy.) 


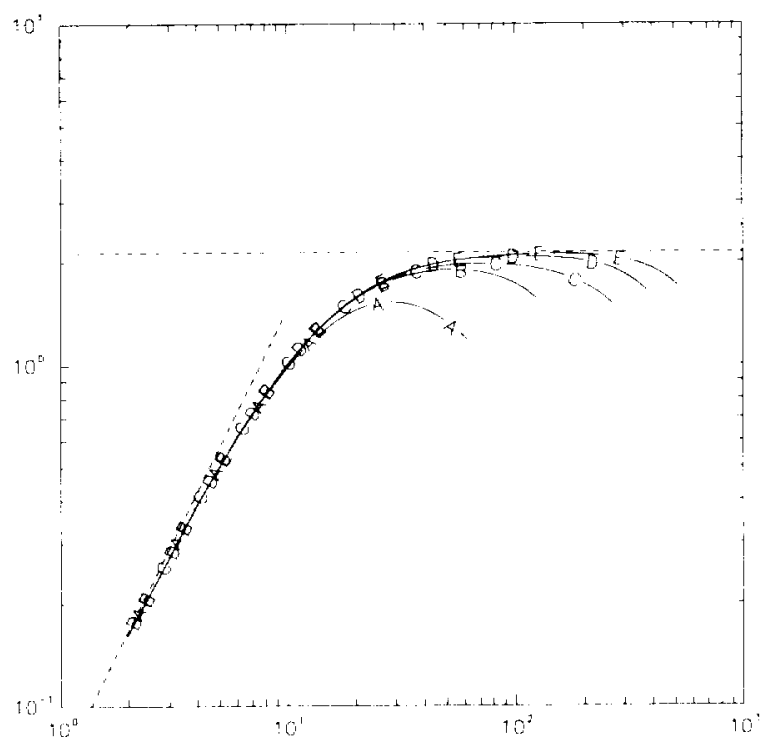

FIG. 6. Kolmogorov scaling of the second-order longitudinal structure function versus $r / e t a$. The horizontal line is at $2.13=(4.02)(0.53) ;$ the sloping dashed line indicates universal behavior of the small scales, as $(1 / 15)(r / \eta)^{4 / 3}$.

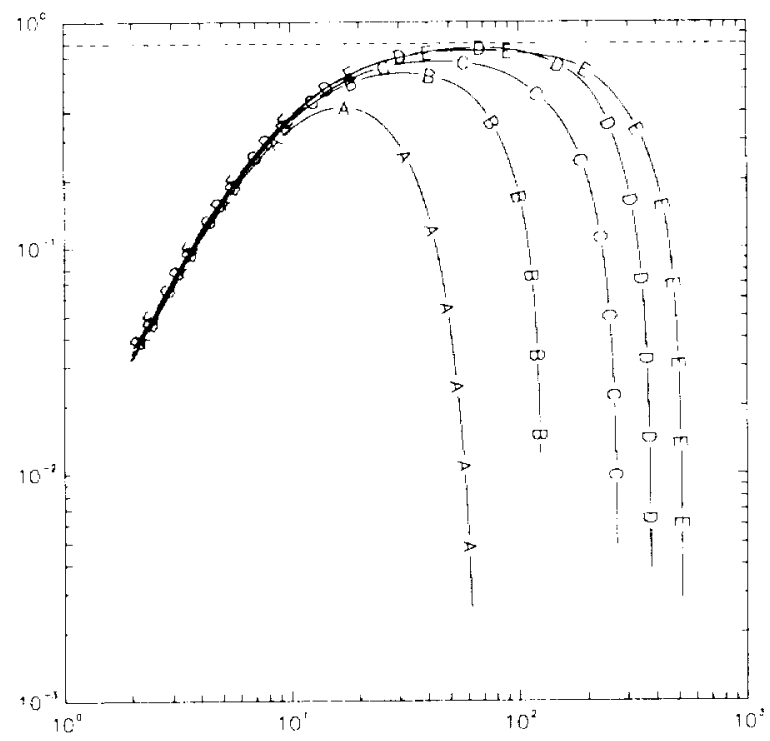

FIG. 7. Kolmogorov scaling of the third order longitudinal structure function versus r/eta. The horizontal line is at 0.8 . 


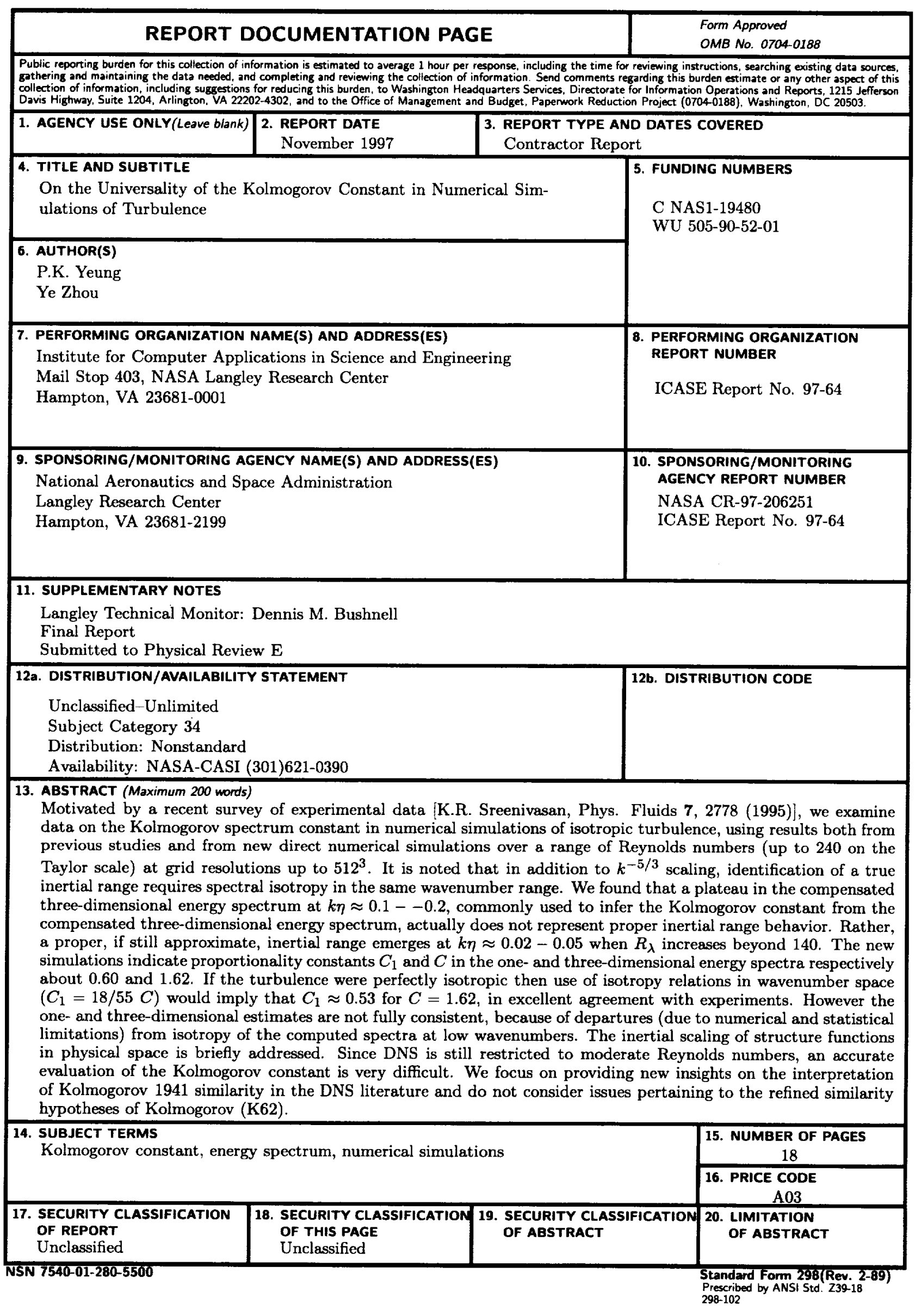

